\title{
FUGA DE TRIAMCINOLONA INTRAVÍTREA A CÁMARA ANTERIOR
}

\section{LEAK OF INTRAVITREAL TRIAMCINOLONE TO THE ANTERIOR CHAMBER}

\author{
BLANCO C ${ }^{1}$, FAGÚNDEZ A ${ }^{2}$, BUZNEGO L ${ }^{1}$, LÓPEZ-MOYA ${ }^{1}$
}

\section{RESUMEN}

Caso clínico: Un hombre de 78 años de edad, fáquico, es sometido a terapia fotodinámica seguida de una inyección de triamcinolona intravítrea. Durante la inyección se observó el paso de una sustancia blanca a cámara anterior. En el examen en la lámpara de hendidura se observó una opacidad blanca en el ángulo inferior de la cámara anterior pero sin signos inflamatorios ni alteración corneal. Discusión: La triamcinolona intraocular se ha usado para el edema macular y diversas enfermedades inflamatorias retinianas. Describimos el paso de esta sustancia a cámara anterior como una complicación muy poco frecuente, probablemente por un defecto de la zónula.

Palabras clave: Acetónido de triamcinolona, inyección intravítrea, complicaciones, presión intraocular, cámara anterior, fáquico.

\begin{abstract}
Case report: A 78-year-old man with phakic eyes underwent photodynamic therapy followed by intravitreal injection of triamcinolone. During the injection a white solution was observed in the anterior chamber. Slit lamp examination revealed white opacities in the inferior anterior chamber angle and neither inflammatory activity nor corneal changes were noticed.

Discussion: Intravitreal triamcinolone has been used for treating macular edema and various retinal vascular and inflammatory diseases. We describe the passage of triamcinolone to the anterior chamber during the injection in a phakic eye as a very unusual adverse effect, observed probably as a result of a zonular defect (Arch Soc Esp Oftalmol 2007; 82: 781-784).
\end{abstract}

Key words: Triamcinolone acetonide, intravitreal injection, complications, intraocular pressure, anterior chamber, phakic.

\section{INTRODUCCIÓN}

Se ha comprobado que la triamcinolona intravítrea puede estabilizar o mejorar la agudeza visual en pacientes con neovascularización coroidea tratados con terapia fotodinámica (TFD) (1).

Están descritas complicaciones secundarias a esta práctica como el aumento de presión intraocular

\footnotetext{
Recibido: 17/10/06. Aceptado: 30/10/07.

Servicio de Oftalmología. Unidad Retina-Vítrea. Complejo Hospitalario Universitario de Albacete. Albacete. España.

1 Licenciado en Medicina.

2 Doctor en Medicina.

Comunicación presentada en el LXXXI Congreso de la S.E.O. (Zaragoza 2005).
}

Correspondencia:

Cristina Blanco Marchite

C/. Alcalde Martínez de la Ossa, 1, 5E

02001 Albacete

España

E-mail: cblancomar@yahoo.es 
(PIO), catarata, desprendimiento de retina, hemorragia vítrea y endoftalmitis infecciosa o aséptica.

\section{CASO CLÍNICO}

Se presenta el caso de un paciente de 78 años de edad, fáquico, diagnosticado de degeneración macular asociada a la edad exudativa (DMAE) en ojo derecho al que se le realiza terapia fotodinámica (TFD). 48 horas después se le inyecta en cámara vítrea $20 \mathrm{mg}$ en $0,1 \mathrm{ml}$ de acetato de triamcinolona obtenida mediante la técnica de sedimentación.

La operación se realizó en el quirófano bajo medidas asépticas. La inyección se hizo con aguja de $27 \mathrm{G}$ a $4 \mathrm{~mm}$ del limbo en el cuadrante temporoinferior ayudado de un blefarostato. Durante el procedimiento, y coincidiendo con un intento de cierre forzado de los párpados, se observó con el microscopio cómo parte de la sustancia lechosa pasaba a cámara anterior.

Se decidió actuar de forma expectante instilando colirio de ciprofloxacino y observando el pulso arterial espontáneo. Al día siguiente el paciente presentaba la triamcinolona precipitada en cámara anterior (fig. 1) y en cámara vítrea sin otros signos inflamatorios. La PIO era de $25 \mathrm{~mm} \mathrm{Hg}$, por lo que se prescribió maleato de timolol al $0,5 \%$ en colirio cada 12 horas.

Se le realizaron controles periódicos en los que se detectó un descenso de la PIO hasta valores normales, una reabsorción progresiva de la triamcinolona

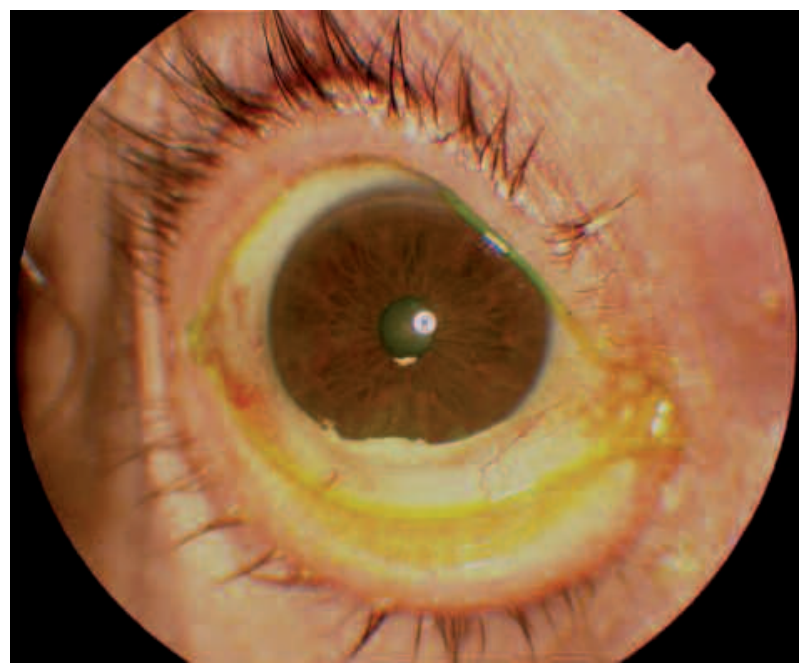

Fig. 1: Depósito de triamcinolona en cámara anterior al día siguiente de la inyección. y la córnea transparente sin reacción inflamatoria. A los dos meses quedaban escasos restos de la sustancia (fig. 2), la PIO era de $17 \mathrm{~mm} \mathrm{Hg}$ sin tratamiento y la biomicroscopía endotelial resultó un recuento celular normal e igual que en el ojo contralateral.

\section{DISCUSIÓN}

Durante décadas se han utilizado los corticoides en oftalmología para suprimir la inflamación intraocular y reducir la extravasación de líquido desde los vasos dañados.

Para evitar los efectos adversos de este medicamento se han investigado distintos métodos de administración.

Las complicaciones descritas tras estas técnicas son diversas. Se puede producir un aumento de la PIO. En la mayoría de los casos este aumento es transitorio y se controla con tratamiento tópico. En los pocos casos en que no es posible el control de la $\mathrm{PIO}$, es preciso una intervención quirúrgica.

Otra de las complicaciones descritas es la aparición de un hipopion en pacientes pseudofáquicos y con mucha menos frecuencia en fáquicos. El cuadro se presenta pasados unos días de la inyección, lo que obliga a hacer un diagnóstico diferencial con la endoftalmitis aguda (2,3). En algunos de estos casos se ha demostrado la existencia de cristales de triamcinolona en el líquido aspirado de cámara anterior, sin células inflamatorias (4).

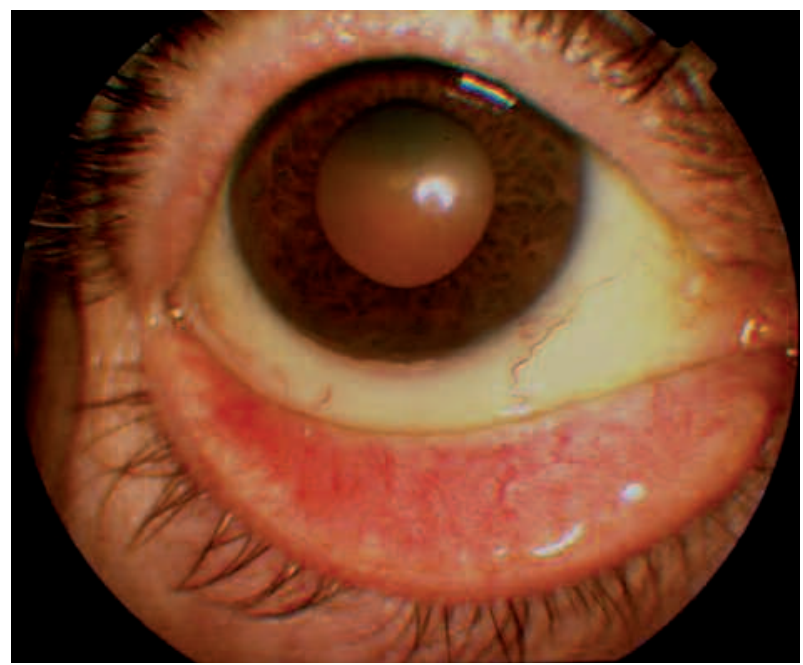

Fig. 2: Escasos restos de triamcinolona en cámara anterior a los dos meses de la inyección. 
Se ha descrito en pacientes pseudofáquicos con rotura de la cápsula posterior, por lo que el pseudohipopion es explicado por el paso de la triamcinolona desde la cavidad vítrea a cámara anterior debido a los movimientos sacádicos oculares. Otras de las teorías que explican este cuadro es una reacción inflamatoria aséptica al excipiente del medicamento (5). Se produce una reacción en cámara anterior sin dolor ni reacción ciliar ni fotofobia, lo que permite descartar un cuadro infeccioso.

Nuestro caso trata de un paciente fáquico en el que durante la inyección intravítrea se observa el paso de la triamcinolona desde la cavidad vítrea a cámara anterior. Se produce la acumulación de triamcinolona en cámara anterior inmediatamente al pinchazo. Creemos que podría ser debido a la existencia de una dehiscencia zonular que permitió el paso de la medicación ayudado por la presión ejercida por el paciente en un intento de cerrar el ojo ante la molestia producida por el pinchazo en esclera.

Tras el seguimiento de nuestro paciente observamos que la cantidad que pasa a cámara anterior no es tóxica para el endotelio corneal.

El paso de triamcinolona a cámara anterior tras inyección en cámara vítrea no sólo se produce en pacientes pseudofáquicos; también puede ocurrir en pacientes que no han sido sometidos a ninguna cirugía.
Se trata de una complicación leve que no produce alteraciones en las estructuras oculares y que se resuelve de forma espontánea con la reabsorción del corticoide de cámara anterior.

A pesar de la no toxicidad del corticoide en estas concentraciones en cámara anterior creemos necesario un seguimiento estricto del proceso.

\section{BIBLIOGRAFÍA}

1. Augustin AJ, Schmidt-Erfurth U. Verteporfin therapy combined with intravitreal triamcinolone in all types of neovascularization due to age-related macular degeneration. Ophthalmology 2006; 113: 14-22.

2. Moshfeghi AA, Scott IU, Flynn HW, Puliafito CA. Pseudohypopion after intravitreal triamcinolone acetonide injection for cystoid macular edema. Am J Ophthalmol 2004; 138: 489-492.

3. Campos VE, Maia A, Muccioli C, Belfort R Jr. Pseudohypopion inmediately after intravitreal injection of triamcinolone acetonide-case reports. Arq Bras Oftalmol 2005; 68: 251-252.

4. Ruiz-Moreno JM, Montero JA, Artola A, Barile S. Anterior chamber transit of triamcinolone after intravitreal injection. Arch Ophthalmol 2005; 123: 129-130.

5. Roth DB, Chieh J, Spirn MJ, Green SN, Yarian DL, Chaudhry NA. Noninfectious endophthalmitis associated with intravitreal triamcinolone injection. Arch Ophthalmol 2003; 121: 1279-1282. 